\title{
Rotation velocities of white dwarfs
}

\section{DA stars with convective atmospheres ${ }^{\star}$}

\author{
C. A. $\operatorname{Karl}^{1}$, R. Napiwotzki ${ }^{1}$, U. Heber ${ }^{1}$, S. Dreizler ${ }^{2,3}$, D. Koester ${ }^{4}$, and I. N. Reid ${ }^{5}$ \\ 1 Dr.-Remeis-Sternwarte, Astronomisches Institut der Universität Erlangen-Nürnberg, Sternwartstr. 7, 96049 Bamberg, \\ Germany \\ e-mail: karl@sternwarte.uni-erlangen.de \\ 2 Universitäts-Sternwarte Göttingen, Geismarlandstr. 11, 37083 Göttingen, Germany \\ 3 Institut für Astronomie und Astrophysik, Sand 1, 72076 Tübingen, Germany \\ 4 Institut für Theoretische Physik und Astrophysik, Universität Kiel, 24098 Kiel, Germany \\ 5 Space Telescope Science Institute, Baltimore, USA
}

Received 9 June 2004 / Accepted 12 November 2004

\begin{abstract}
The sharp H $\alpha$ NLTE line cores of hydrogen-rich (DA) white dwarfs allow their projected rotational velocities to be determined. High resolution optical spectra of 22 stars obtained with the Keck I telescope are matched by synthetic spectra computed from a grid of NLTE model atmospheres. In this paper, the third in a series on white dwarf rotation, we concentrate preferentially on DA white dwarfs with convective atmospheres, i.e. with $T_{\text {eff }}<14000 \mathrm{~K}$. Previous analyses found DA white dwarfs hotter than $14000 \mathrm{~K}$ to be very slow rotators and rarely show any spectroscopically detectable rotation. For 19 of our programme stars we were able to derive projected rotational velocities or upper limits. No rotation could be detected for seven stars in our sample. However twelve stars show significant line broadening. In the case of the ZZ Ceti star G 117-B15A, the observed $\mathrm{H} \alpha$ line profile cannot be matched by a rotationally broadened profile as its line core is too narrow. Combining our results with those from two similar studies, we have obtained information on the rotation or other line broadening mechanisms (such as caused by magnetic fields) of 56 DA white dwarfs. The fraction of rotating DA white dwarfs whose line profiles can be matched for a vanishing projected rotation velocity is high for hot white dwarfs with radiative atmospheres (25 out of 28). Amongst the cool white dwarfs with presumedly convective atmospheres, only for a few stars (8 out of 22) has no additional line broadening to be invoked to explain their observed $\mathrm{H} \alpha$ line profiles. We conjecture that the physics of $\mathrm{H} \alpha$ line formation in convective DA white dwarf atmospheres is not yet sufficiently well understood and additional observational and theoretical efforts have to be made.
\end{abstract}

Key words. stars: white dwarfs - stars: variables: general - stars: rotation - stars: convection - stars: atmospheres

\section{Introduction}

White dwarfs are the compact remnants of low and intermediate mass stars $\left(M<8 M_{\odot}\right)$. Due to the conservation of angular momentum white dwarfs should be very fast rotators, if a significant fraction of the angular momentum of the progenitor stars were preserved. Although the spectral lines of white dwarfs are generally very broad, the existence of sharp NLTE cores of the hydrogen $\mathrm{H} \alpha$ line in DA white dwarfs allows one to determine (projected) rotational velocities $v \sin i$ for these stars.

Several groups (Greenstein et al. 1977; Pilachowski \& Milkey 1984, 1987; Koester \& Herrero 1988; Heber et al. 1997; Koester et al. 1998) applied this method to measure rotational

^ Based on observations at the W. M. Keck Observatory, which is operated by the Californian Association for Research in Astronomy for the California Institute of Technology and the University of California. velocities of 53 white dwarfs. A compilation of the existing sample is provided by Koester et al. (1998).

For most white dwarfs, only upper limits on the rotational velocities of the order of $20-40 \mathrm{~km} \mathrm{~s}^{-1}$ are available. Thus the general conclusion is that white dwarfs are slow rotators. This result is supported by astereoseismological investigations of pulsating white dwarfs. Rotation periods of the very hot white dwarfs PG 1159-035 (Winget et al. 1991) and PG 0122+200 (O'Brien et al. 1996), the DB star GD 358 (Winget et al. 1994) and ZZ Ceti stars (e.g. Kleinman et al. 1998) have been determined from the rotational splitting of oscillation modes. The determined periods of the stars range from 1.17 to 1.6 days, corresponding to rotational velocities of $\approx 1 \mathrm{~km} \mathrm{~s}^{-1}$. Polarization measurements of magnetic white dwarfs also indicate that most of these objects are very slow rotators (see Table 1 in Wickramasinghe \& Ferrario 2000). However, magnetic white dwarfs may not be representative of the entire class of white 
Table 1. Atmospheric parameters, masses, radii and projected rotational velocities of the programme stars. Line profiles that cannot be matched at all by rotational profiles are marked as peculiar (see text).

\begin{tabular}{|c|c|c|c|c|c|c|c|c|}
\hline WD & White dwarf & $\begin{array}{l}T_{\text {eff }} \\
{[\mathrm{K}]}\end{array}$ & $\begin{array}{l}\log g \\
{\left[\frac{\mathrm{cm}}{\mathrm{s}^{2}}\right]}\end{array}$ & $\begin{array}{c}M \\
{\left[M_{\odot}\right]}\end{array}$ & $\begin{array}{c}R \\
{\left[R_{\odot}\right]}\end{array}$ & Ref. & $\begin{array}{c}v \sin i\left[\mathrm{~km} \mathrm{~s}^{-1}\right] \\
1 \sigma\end{array}$ & $\begin{array}{c}v \sin i\left[\mathrm{~km} \mathrm{~s}^{-1}\right] \\
3 \sigma\end{array}$ \\
\hline \multicolumn{9}{|c|}{ Parameters from spectral analyses: } \\
\hline $0220+222$ & G $94-$ B5B & $15840 \pm 364$ & $7.95 \pm 0.07$ & 0.561 & 0.0131 & 1 & $<9$ & $<20$ \\
\hline $0251-008$ & LP $591-117$ & $7700 \pm 178$ & $8.05 \pm 0.07$ & 0.624 & 0.0124 & 8 & $24_{-5}^{+4}$ & $24_{-12}^{+10}$ \\
\hline $0518+333$ & G $86-B 1 B$ & $9140 \pm 210$ & $8.30 \pm 0.07$ & 0.767 & 0.0103 & 1 & $<7$ & $<17$ \\
\hline $0810+234$ & GD 295 B & $15220 \pm 360$ & $7.99 \pm 0.07$ & 0.579 & 0.0127 & 1 & $<16$ & $<26$ \\
\hline $0836+201$ & LB 390 & $16630 \pm 382$ & $8.04 \pm 0.07$ & 0.624 & 0.0129 & 7 & $66_{-14}^{+14}$ & $66_{-59}^{+46}$ \\
\hline $0913+442$ & G $116-16$ & $8750 \pm 201$ & $8.29 \pm 0.07$ & 0.759 & 0.0103 & 1 & \multicolumn{2}{|c|}{ peculiar } \\
\hline $0921+354$ & $\mathrm{G} 117-\mathrm{B} 15 \mathrm{~A}^{a}$ & $12830 \pm 295$ & $7.92 \pm 0.07$ & 0.537 & 0.0133 & 1 & $36_{-5}^{+7}$ & $36_{-17}^{+23}$ \\
\hline $1147+255$ & LP 375 - 51 & $10260 \pm 236$ & $8.12 \pm 0.07$ & 0.651 & 0.0116 & 1 & $24_{-7}^{+7}$ & $24_{-15}^{+19}$ \\
\hline $1706+332$ & G 181 - B5B & $13600 \pm 312$ & $7.79 \pm 0.07$ & 0.470 & 0.0145 & 1 & $23_{-4}^{+3}$ & $23_{-10}^{+9}$ \\
\hline $1716+020$ & Wolf $672 \mathrm{~A}$ & $13620 \pm 313$ & $7.79 \pm 0.07$ & 0.470 & 0.0145 & 1 & $<11$ & $<15$ \\
\hline $1743-132$ & G 154 - B5B & $13950 \pm 320$ & $7.71 \pm 0.07$ & 0.431 & 0.0165 & 1 & $28_{-4}^{+3}$ & $28_{-9}^{+8}$ \\
\hline $2047+809$ & LP 25 - 436 & $8840 \pm 203$ & $8.50 \pm 0.07$ & 0.916 & 0.0087 & 1 & \multicolumn{2}{|c|}{ peculiar } \\
\hline $2253-081$ & G $156-64$ & $7160 \pm 165$ & $8.43 \pm 0.07$ & 0.857 & 0.0093 & 1 & $36_{-7}^{+15}$ & $36_{-17}^{+27}$ \\
\hline $2258+406$ & G 216 - B14B & $9860 \pm 226$ & $8.20 \pm 0.07$ & 0.701 & 0.0110 & 1 & $20_{-5}^{+6}$ & $<42$ \\
\hline \multicolumn{9}{|c|}{ Parameters from photometry and trigonometric parallaxes: } \\
\hline $0518+333$ & G $86-\mathrm{B} 1 \mathrm{~B}$ & $7780 \pm 180$ & $7.14 \pm 0.24$ & 0.24 & 0.022 & 6 & $10_{-9}^{+5}$ & $<20$ \\
\hline $0913+442$ & G $116-16$ & $8490 \pm 220$ & $8.19 \pm 0.17$ & 0.71 & 0.011 & 6 & \multicolumn{2}{|c|}{ peculiar } \\
\hline $1147+255$ & LP $375-51$ & $9790 \pm 270$ & $7.92 \pm 0.23$ & 0.55 & 0.013 & 6 & $26_{-12}^{+7}$ & $26_{-17}^{+19}$ \\
\hline $1716+020$ & Wolf $672 \mathrm{~A}$ & $13470 \pm 900$ & $7.92 \pm 0.23$ & 0.67 & 0.015 & 6 & $<7$ & $<10$ \\
\hline $2253-081$ & G $156-64$ & $6770 \pm 180$ & $8.41 \pm 0.19$ & 0.86 & 0.010 & 6 & $52_{-15}^{+16}$ & $52_{-24}^{+29}$ \\
\hline \multicolumn{9}{|c|}{ Parameters from photometry and gravitational redshifts: } \\
\hline $0030+444$ & G $172-4$ & $10440 \pm 240$ & $8.02 \pm 0.07$ & 0.578 & 0.0123 & 3 & $27_{-4}^{+5}$ & $27_{-12}^{+14}$ \\
\hline $0628-020$ & LP $600-42$ & $9000 \pm 207$ & $7.88 \pm 0.07$ & 0.532 & 0.0139 & 2 & \multicolumn{2}{|c|}{ peculiar } \\
\hline $0807+484$ & LP $163-121$ & $7000 \pm 161$ & $8.01 \pm 0.07$ & 0.596 & 0.0126 & 2 & $<18$ & $<53$ \\
\hline $0906+296$ & LP $313-16$ & $10000 \pm 230$ & $7.86 \pm 0.07$ & 0.524 & 0.0141 & 2 & $41_{-5}^{+6}$ & $41_{-13}^{+15}$ \\
\hline $1240+754$ & G 255 - B18A & $12000 \pm 276$ & $7.96 \pm 0.07$ & 0.553 & 0.0129 & 2 & $30_{-10}^{+7}$ & $<62$ \\
\hline $1510+566$ & LP 135 - 154 & $9280 \pm 213$ & $7.98 \pm 0.07$ & 0.626 & 0.0134 & 3 & $<11$ & $<19$ \\
\hline $1710+683$ & LP 70 - 172 & $6825 \pm 90$ & $7.94 \pm 0.07$ & 0.559 & 0.0133 & 4 & $72_{-10}^{+11}$ & $72_{-23}^{+27}$ \\
\hline $1211+392$ & LP $216-74$ & $18800 \pm 1600$ & $7.7 \pm 0.22$ & & & 5 & $<18$ & $<53$ \\
\hline
\end{tabular}

${ }^{a}$ ZZ Ceti star, the inermost line core is not matched well (see text and Fig. 4).

References:

1 - Bergeron et al. (1995a);

2 - Reid (1996);

$3-T_{\text {eff }}$ and $\log g$ from Weidemann \& Koester (1984) and masses from Reid (1996);

$4-T_{\text {eff }}$ derived from photometry and $\log g$ from mass and gravitational redshift (Reid 1996);

5 - Kidder (priv. com.), based on $U B V$ photometry of Kidder et al. (1991);

6 - Bergeron et al. (2001);

7 - Claver et al. (1998);

8 - $T_{\text {eff }}$ derived from the $\mathrm{H}_{\alpha}$ line profile and $\log g$ from mass and redshift (Reid 1996).

dwarfs because the evolution of angular momentum could have been influenced by the strong magnetic fields.

These low rotational velocities indicate that the angular momentum of the stellar cores is efficiently transported to the stellar surface and carried away by the mass lost during the course of stellar evolution. However, the emerging picture is challenged by the puzzling results presented in the recent investigation of Koester et al. (1998, henceforth Paper II). The authors analyzed the $\mathrm{H} \alpha$ NLTE cores of 25 DA white dwarfs spanning the temperature range $7000 \mathrm{~K}$ to $30000 \mathrm{~K}$. Most white dwarf spectra were compatible with zero rotational velocity. However, all three ZZ Ceti variables in that sample showed significant extra broadening of the observed line cores. Rotational broadening profiles, however, did not match the observations well.

Therefore Koester et al. (1998) argue that the observed broadening is not due to rotation and discussed velocity fields caused by pulsation and convection but find them to be far too small to explain the observed spectra. Recently it became possible to measure radial velocity variations of ZZ Ceti stars (Van Kerkwijk et al. 2000; Kotak et al. 2002b; Thompson et al. 2003), which confirmed that the amplitudes indeed are as low as predicted. Even if the broadening were caused by rotation it would be difficult to understand how white dwarfs could spinup when entering the instability strip during their evolution and spin-down again after leaving it. 
It is obvious that more data are needed to improve the statistics near the ZZ Ceti strip and to pin down the temperature range in which this phenomenon occurs. This will also help to identify the underlying mechanism. In this article we present the results of the analysis of the NLTE cores of 22 DA white dwarfs covering the temperature range from $6800 \mathrm{~K}$ to $20000 \mathrm{~K}$. This is an extension of our previous investigation (Heber et al. 1997, henceforth Paper I) to lower temperatures.

The analysis is based on the spectra obtained by Reid (1996) with the HIRES spectrograph of the Keck I telescope. Reid (1996) used these spectra to measure the gravitational redshift of white dwarfs in common proper motion pairs and open clusters. In our previous analysis (Paper I) we modeled the line profiles of 13 hotter stars $\left(T_{\text {eff }} \gtrsim 14000 \mathrm{~K}\right)$ of this sample and determined upper limits on $v \sin i$. No significant rotation was detected in any case. The analysis of the remaining 22 useful spectra of this sample is presented in this paper.

This paper is organized as follows. In Sect. 2 we summarize the available atmospheric parameters and derive stellar masses. In Sect. 3 the spectral analysis of $\mathrm{H} \alpha$ profiles is described and the resulting projected rotational velocities are presented in Sect. 4. The latter are discussed in the concluding Sect. 5.

\section{Atmospheric parameters and stellar masses}

The atmospheric parameters $T_{\text {eff }}$ and $\log g$ need to be known in order to compute synthetic $\mathrm{H} \alpha$ line profiles.

Atmospheric parameters $\left(T_{\text {eff }}, \log g\right)$ determined spectroscopically were preferred when available. Values for 12 stars were taken from Bergeron et al. (1995a) and one from Claver et al. (1998). These authors used a mass-radius relation and the gravity to determine radii and masses. We adopted the masses as given in the cited papers.

For five of those (mostly cooler) stars, atmospheric parameters have also been obtained from photometry and trigonometric parallaxes (Bergeron et al. 2001). These values were used for intercomparison (see below).

For all other programme stars we had to rely on photometric temperature estimates. Effective temperatures of two stars were derived from multicolour photometry by Weidemann \& Koester (1984). For the remaining stars we have either determined the effective temperature from photometry listed in the catalog of McCook \& Sion (1998) using the colour calibrations of Bergeron et al. (1995b) or we adopted the temperatures derived by Reid (1996).

Gravitational redshifts were combined with the massradius-relation to derive masses and radii (Reid 1996). We then calculated the surface gravities from the latter.

The error budget was calculated as described in Napiwotzki et al. (1999) for the surface gravities of all stars and for most of the temperatures. Errors for photometric temperatures were estimated from the errors of the colors.

The temperatures range from 6800 to $20000 \mathrm{~K}$ and $\log g$ from 7.70 to 8.50 . Only four stars (WD $0220+222$, WD 0810+234, WD 0836+201 and WD 1211+392) have effective temperatures exceeding $14000 \mathrm{~K}$. Their atmospheres are therefore radiative. The masses lie between 0.43 and $0.92 M_{\odot}$.
The parameters of the observed stars used for the subsequent analysis are summarized in Table 1.

\subsection{Individual objects}

Some stars deserve a more detailed discussion because the parameter determination was not straight forward.

G 86 - B1B = WD 0518+333: Bergeron et al. (2001) mark this star as possible double degenerate. The atmospheric parameters derived from spectroscopy (Bergeron et al. 1995a) differ considerably from those derived from photometry and trigonometric parallax (Bergeron et al. 2001). Since it is unclear which set of parameters should be prefered, we carried out the analsyis of the H $\alpha$ profile with both sets. Reid (1996) noticed that the $\mathrm{H} \alpha$ line core seems to be disturbed Since the star was observed near dawn, he considered the emission spike as an artefact from residual night sky emission. We inspected Reid's spectrum but could not find any other residual night sky emission throughout the entire spectrum. We noticed, however, that the line core of $\mathrm{H} \beta$ displays a slight asymmetry. The $\mathrm{H} \alpha$ profile is similar to that of WD $1845+019$ (see Fig. 2 of Maxted \& Marsh 1999). In this case the emission is due to a cool companion star in a long orbit around the white dwarf. This scenario could also be valid for WD $0518+333$ and might explain why different methods of analysis give such disparate results.

The low gravity derived by Bergeron et al. (2001) is not covered by our model grid. Therefore some extrapolation was inevitable. Despite the large differences in atmospheric parameters the two resulting limits on the projected rotation velocity are quite similar (see Table 1 ).

G 117-B15A = WD 0921+354 is a known ZZ Ceti which has been studied in great detail (see Kepler et al. 2000, and references therein).

LP 375-51 = WD 1147+255 and G 216-B14B = WD 2258+406 lie near the red edge of the ZZ Ceti instability strip but are non-variable (Giovannini et al. 1998). The atmospheric parameters derived from spectroscopy (Bergeron et al. 1995a) are consistent with those derived from photometry and trigonometric parallax (Bergeron et al. 2001). LP 135-154 = WD 1510+566 and G 172-4 = WD 0030+444: $T_{\text {eff }}$ and $\log g$ were derived by Weidemann \& Koester (1984) from analysis of multichannel photometry.

Wolf $672 \mathrm{~A}=$ WD 1716+020 lies near the blue edge of the ZZ Ceti instability strip but is non-variable (Giovannini et al. 1998). The effective temperature derived from spectroscopy (Bergeron et al. 1995a) is consistent with that derived from photometry and trigonometric parallax (Bergeron et al. 2001), whereas the spectroscopic gravity is somewhat higher than derived from the trigonometric parallax.

G 154-B5B = WD 1743-132 The projected rotation velocity has also been determined in Paper II.

G 156 - 64 = WD 2253-081 The atmospheric parameters derived from spectroscopy (Bergeron et al. 1995a) are consistent with those derived from photometry and trigonometric parallax (Bergeron et al. 2001).

LP 591-117 = WD 0251-008 We used the photometric data found in the Mc Cook \& Sion catalogue (1999) and the color 


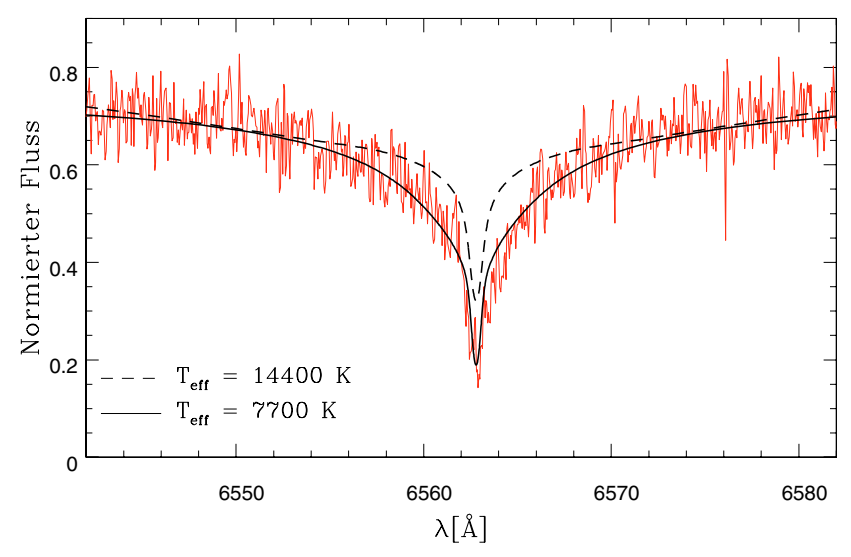

Fig. 1. $\mathrm{H} \alpha$ line profile fit for WD 0251-008. The observed profile is compared to non-rotating synthetic line profiles calculated at $14400 \mathrm{~K}$ as derived from photometry (dashed line) and $7700 \mathrm{~K}$ as we found from the $\mathrm{H}_{\alpha}$ line fit (solid line).

calibrations from Bergeron et al. (1995) to derive the effective temperature. However, the temperatures calculated from the Broadband $B-V$ and $U-B(15200 \mathrm{~K}$ and $160000 \mathrm{~K}$ resp.) differs from the temperature derived using Strömgren $b-y(11900 \mathrm{~K})$. The mean temperature of $14400 \mathrm{~K}$ cannot reproduce the $\mathrm{H}_{\alpha}$ line profile.

Reid (1995) estimated a lower effective temperature of $T_{\text {eff }}=9000 \mathrm{~K}$ while Silvestri et al. (2001) found $6453 \mathrm{~K}$. In order to clarify these large differences we fitted the $\mathrm{H}_{\alpha}$ line wings in our Keck spectrum (neglecting the line core) using the model grid described in Sect. 3.1. From the best fit we obtained $7700 \mathrm{~K}$ (see Fig. 1, which is close the mean value of Reid and Silvestri et al. To derive $v \sin i$ we used this temperature for the further analysis of the $\mathrm{H}_{\alpha}$ line core and adopted $\log g=8.05$ as computed from Reid (1995).

\section{Model atmospheres and spectral analysis}

\subsection{Model atmospheres}

In Paper I we used self consistent, line blanketed NLTE model atmospheres which, however, neglected convection. Therefore we had to restrict our analysis to white dwarfs hotter than $14000 \mathrm{~K}$. The atmospheres are radiative for $T_{\text {eff }} \gtrsim 16000 \mathrm{~K}$. Below this and depending on the choice of gravity the atmospheres become convective, however the convection is inefficient and has almost no influence on the atmospheric structure as long as $T_{\text {eff }} \gtrsim 12500 \mathrm{~K}$.

In Paper II LTE models were used that account for convection (mixing length theory using ML2/ $\alpha=0.6$, see Paper II for details) to determine the temperature - pressure stratification of the atmosphere. With this stratification kept fixed, the line formation was computed in NLTE, solving the rate equations for the occupation number at each depth.

The dominant continuum absorption mechanism in cool DA is $\mathrm{H}^{-}$absorption which is also not included in the NLTE calculations. Therefore in Paper II $\mathrm{H}^{-}$opacity was included as a background absorption and calculated in the LTE approximation.
The model grid used for our analysis is the same as used in Paper II. It covers the parameter range $T_{\text {eff }}=7000 \mathrm{~K}$ to $25000 \mathrm{~K}$ in steps of $1000 \mathrm{~K}$ and $\log g=7.5,8.0$ and 8.5.

\subsection{Analysis technique}

The technique for the analysis of the $\mathrm{H} \alpha$ line profiles is described in Paper I. Briefly, the synthetic $\mathrm{H} \alpha$ line profiles are interpolated from a grid of model spectra (convolved with the instrument profile, a Gaussian with $0.14 \AA F W H M$ ). Only the innermost part (about $\pm 20 \AA$ ) of the line profile was used for comparison with observations. Wavelength scales were adjusted by cross correlating the observed and the synthetic spectra. The rotational profile (in the notation of Gray 1992) was used for the line broadening of the synthetic spectra and a limb darkening coefficient of $\epsilon=0.15$ following Pilachowski et al. (1984) was adopted. Since no continuum point can be found close to the line cores, the observation and the synthetic profiles were adjusted at some preselected wavelength points outside of the spectral range used in the fitting process.

For each star a grid of synthetic spectra was produced by varying $v \sin i$ in steps of $1 \mathrm{~km} \mathrm{~s}^{-1}$. Four additional sets of synthetic spectra per star were obtained by varying the effective temperature and surface gravity within their error limits.

These sets were matched to the observed spectra by calculating the $\chi^{2}$ deviations using a modified version of Bergeron's and Saffer's program (Saffer et al. 1994). Subtracting the minimum $\chi^{2}$ we obtain a relation of $\Delta \chi^{2}$ with $v \sin i$ for each combination of $T_{\text {eff }}$ and $\log g$ (see Figs. 2 and 3). $1 \sigma$ and $3 \sigma$ confidence level are read from these plots at $\Delta \chi^{2}=1$ and $\Delta \chi^{2}=9$, respectively. Previous analyses have used either a $1 \sigma$ (e.g. Paper II) or a $3 \sigma$ criterion (Paper I). Therefore we give both values for our programme stars in Table 1. For the purpose of comparison we have recalculated the $1 \sigma$ limits for the stars of Paper I and list them in Table 2.

\section{Projected rotational velocities}

Projected rotational velocities for 19 of our 22 programme stars have been determined. For three stars (WD 0628-020, WD 0913+442 and WD 2047+809) no reasonable fit could be obtained (cf. Sect. 4.1). Resultes are listed in Table 1 were errors at the $1 \sigma$ and $3 \sigma$ level are given.

For seven stars of our sample the best fit was achieved for vanishing rotation ( $v \sin i=0$ within the $1 \sigma$ error limits). The quality of the fits are demonstrated for four non-rotating stars in Fig. 2; spectra of different noise levels are displayed.

Another twelve stars showed extra broadening. As can be seen from Fig. 3, line profiles broadened by rotational profiles with $v \sin i$ between $20 \mathrm{~km} \mathrm{~s}^{-1}$ and $72 \mathrm{~km} \mathrm{~s}^{-1}$ match the observations well. Strong magnetic fileds can broaden lines due to Zeeman splitting as well. Koester et al. (1998) discovered magnetically broadened line profiles for some of their objects. However, no hints of Zeeman triplets can be found among our spectra, not even "flat bottom" line profiles (cf. Figs. 7 to 9 in Paper II). Moreover, from the most extreme line broadening of $0.35 \AA$ (WD 1710+683) we deduce an upper limit for the 

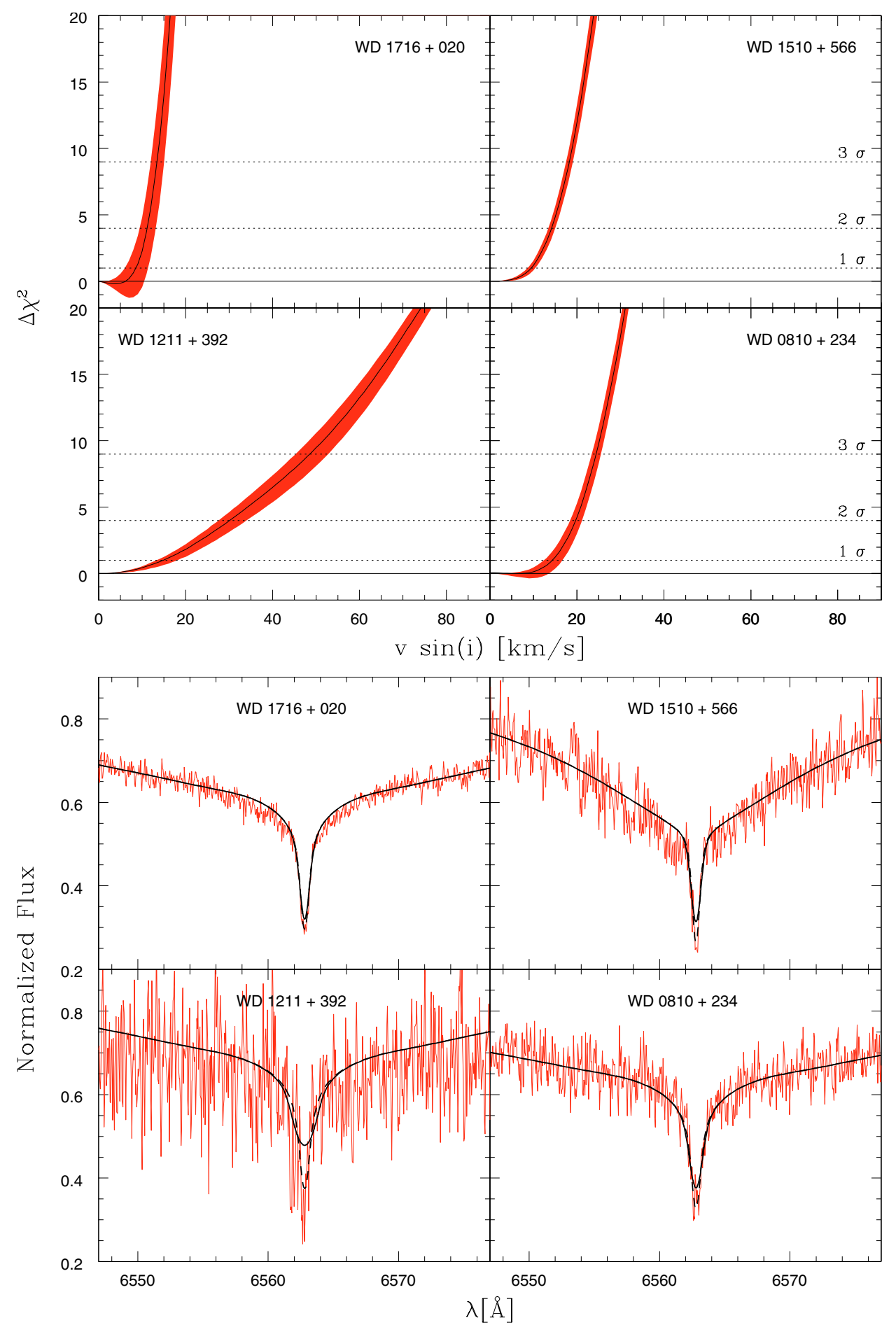

Fig. 2. $\mathrm{H} \alpha$ line profile fits and upper limits for the projected rotational velocities of four DA stars. For each star the upper panel displays the run of $\Delta \chi^{2}$ with $v \sin i$ from which the upper limits were read off, the lower panel displays the match of the observed profile by a line profile without rotation (dashed line) and for the maximum $v \sin i$ deduced from the $3 \sigma$ limit (full line).

magnetic fields of $17.5 \mathrm{kG}$ only. Thus we exclude any significant broadening due to magnetic fields.

\subsection{Individual objects}

WD 0921+354: the fit for this ZZ Ceti variable, however, is peculiar. The formal best fit is obtained for $v \sin i=36 \mathrm{~km} \mathrm{~s}^{-1}$ (upper panel of Fig. 4). However, inspection of the line profile fit reveals that the observed line core is narrower than predicted by the synthetic line profile. This is demonstrated in Fig. 5, where we compare the line profile fit for WD $0921+354$ to those of the non-rotating star WD $1716+020$ and the rotating star WD $1706+333$. We have to conclude that the line broadening of the ZZ Ceti star WD $0921+354$ cannot be due to rotation, but must be caused by an unknown physical mechanism. The same conclusion was reached in Paper II for three ZZ Ceti stars. 

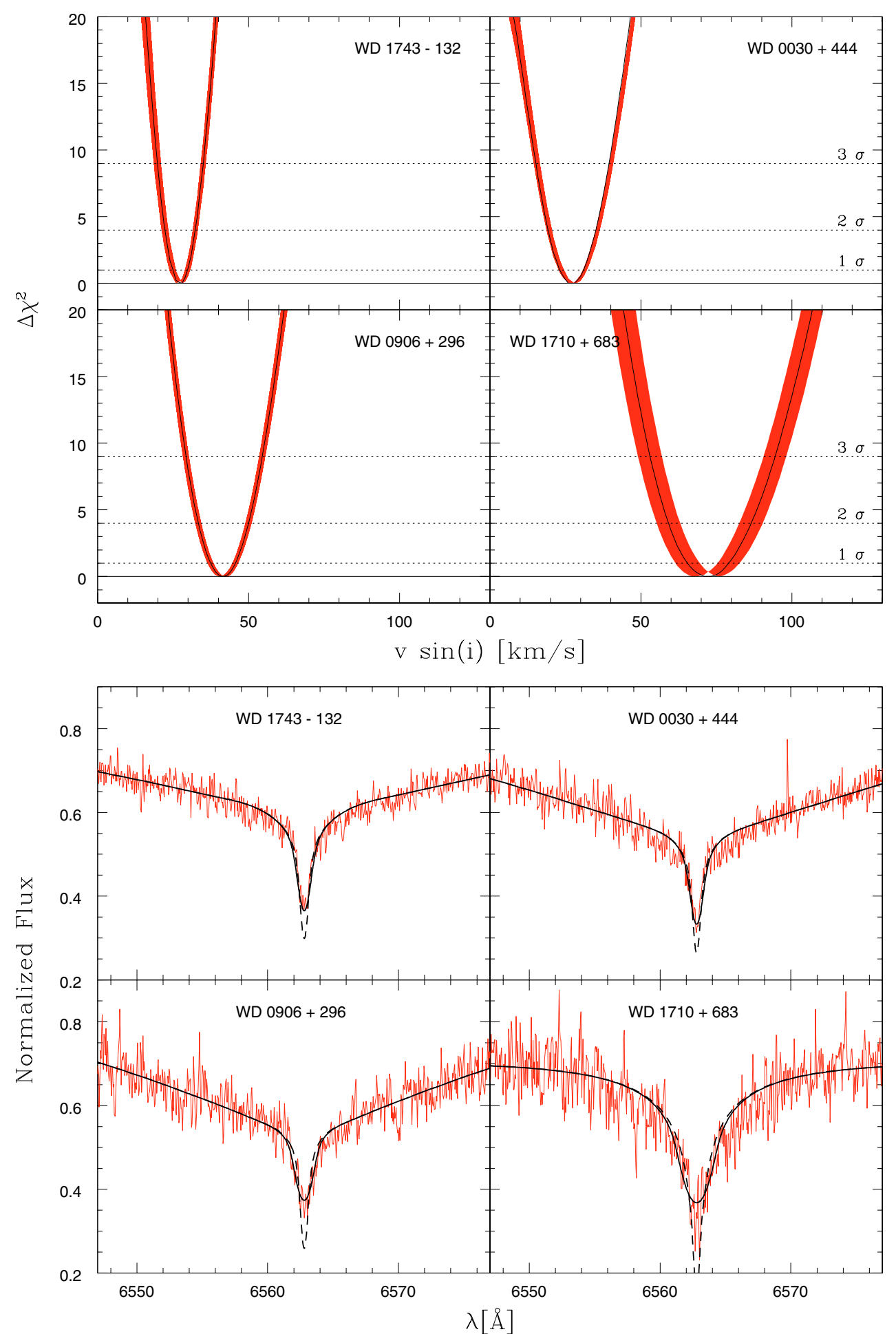

Fig. 3. H $\alpha$ line profile fits and best fits for the projected rotational velocities of four DA stars. For each star the upper panel displays the run of $\Delta \chi^{2}$ with $v \sin i$ from which the best fit projected rotational velocity is read off, the lower panel displays the match of the observed profile by a line profile without rotation (dashed line) and for the best fit $v \sin i$ (full line).

WD $0518+333$ is a special case among our program stars. Two parameter sets had to be used. There is no evidence for rotation at the $3 \sigma$ level, whereas if we apply the $1 \sigma$ criterion and use the parameters derived by Bergeron et al. (2001) the star would be marginally rotating.

WD 1240+754 and WD 2258+406: rotation of these stars is detected at a $1 \sigma$ level $\left(v_{\text {rot }} \sin i=30 \mathrm{~km} \mathrm{~s}^{-1}\right.$ and $20 \mathrm{~km} \mathrm{~s}^{-1}$ resp. $)$ but not at the $3 \sigma$ level for which only rather high upper limits of $62 \mathrm{~km} \mathrm{~s}^{-1}$ and $42 \mathrm{~km} \mathrm{~s}^{-1}$ can be quoted.

WD 0628-020, WD 0913+442 and WD 2047+809 show broadened lines, too. However, in these cases the broadening is very different from a rotational profile as can be seen from 
Table 2. Upper limits $(1 \sigma$ and $3 \sigma$ ) for the projected rotational velocities of the stars from Paper I. $1 \sigma$ limits have not been published previously.

\begin{tabular}{lccc}
\hline \hline WD & White dwarf & $v \sin i\left(\mathrm{~km} \mathrm{~s}^{-1}\right)$ & $v \sin i\left(\mathrm{~km} \mathrm{~s}^{-1}\right)$ \\
& & $1 \sigma$ & $3 \sigma$ \\
\hline $0352+096$ & HZ 4 & $<13$ & $<21$ \\
$0406+169$ & LB 227 & $<20$ & $<28$ \\
$0421+162$ & VR 7 & $<8$ & $<18$ \\
$0425+168$ & VR 16 & $<18$ & $<26$ \\
$0431+125$ & HZ 7 & $15_{-5}^{+7}$ & $<35$ \\
$0438+108$ & HZ 14 & $<14$ & $<28$ \\
$0836+197$ & LB 5893 & $<22$ & $<43$ \\
$0837+199$ & LB 393 & $<9$ & $<29$ \\
$0413-077$ & 40 Eri B & $<1$ & $<8$ \\
$0726+393$ & LP207-7 & $<7$ & $<18$ \\
$1143+321$ & G148-7 & $<2$ & $<12$ \\
$1314+293$ & HZ43 & $<11$ & $<29$ \\
$1911+135$ & G142-B2B & $<2$ & $<9$ \\
\hline
\end{tabular}
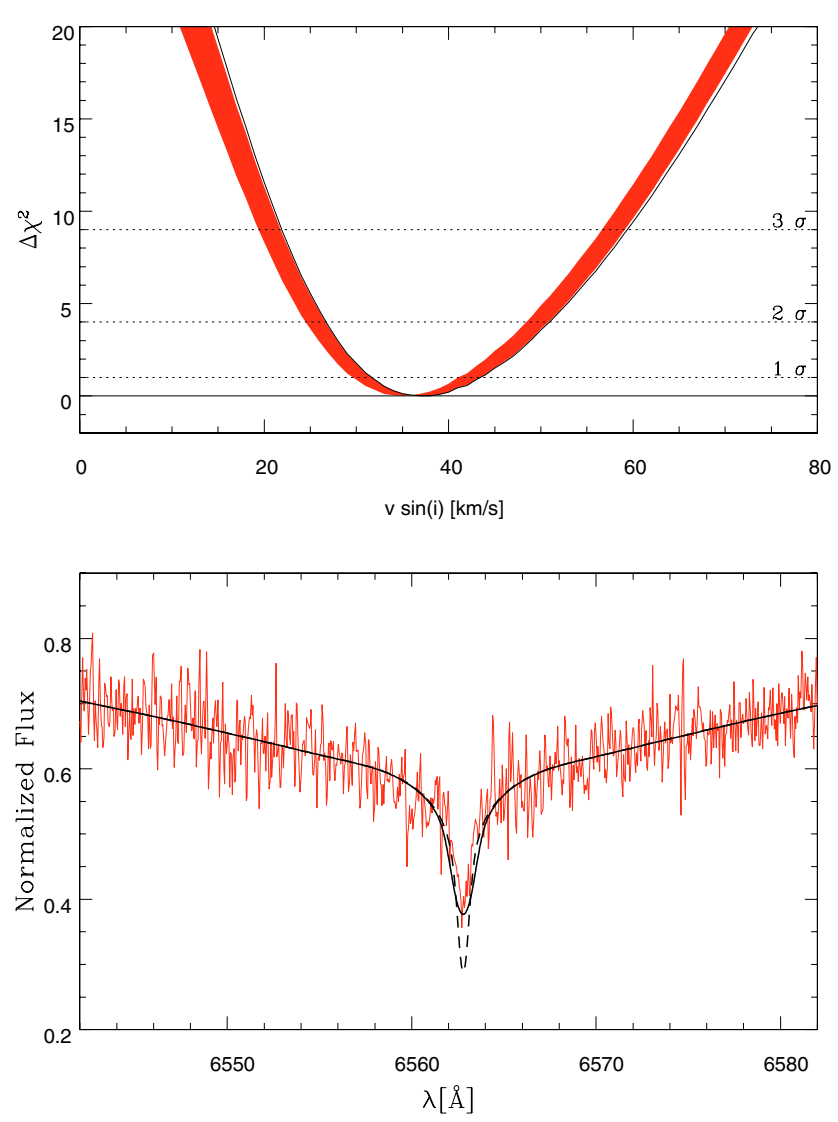

Fig. 4. $\mathrm{H} \alpha$ line profile fits for the ZZ Ceti variable WD 0921+354 (G117-B15A). The upper panel displays the run of $\Delta \chi^{2}$ with $v \sin i$ from which the best fit and error limits are read off, the lower panel displays the match of the observed profile by a line profile without rotation (dashed line) and for the best fit $v \sin i$ (full line).

Fig. 6 in the case of WD 0628-020. While a sharp line core can be reproduced by a synthetic line profile and vanishing rotation, the near line wings are far too broad. The formal best fit gives a very poor fit to the observation.

To improve the fit quality we changed the stellar parameters within larger error limits than indicated in Table 1. We found

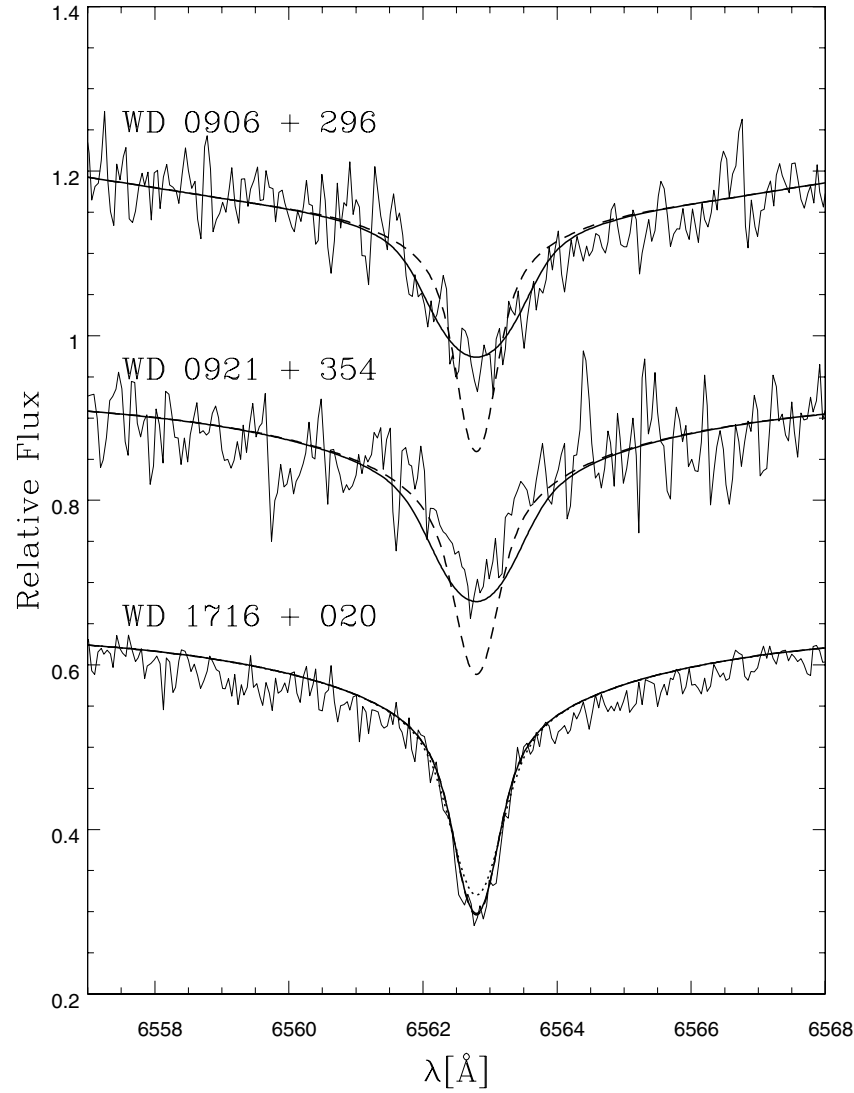

Fig. 5. $\mathrm{H} \alpha$ line profile fits for the ZZ Ceti variable WD $0921+354$ (middle) compared to those of the rotating star WD 1706+354 (top) and the non-rotating star WD 1716+020 (bottom). The line core of G117-B15A cannot be matched by a rotational profile.

that $\log g$ hardly influences the shape of the line profile, while a decrease of $T_{\text {eff }}$ by $\approx 1000 \mathrm{~K}$ will reasonably improve the fit of the core and the near line wings. In this region the shape of the observed line profile is now very similar to the synthetic profile, whereas the outer line wings are not well matched, as can be seen from Fig. 7 .

Since the innermost line cores of these "peculiar" objects show neither the typical Zeeman splitting nor "flat bottom" profiles, we exclude magnetic fields as the reason for this puzzling effect. An anomalous limb darkening can also be excluded since the rotational profile is insensitive to the variation of this coefficient (see Pilachowski et al. 1984). Thus we have to conclude that their line profiles cannot be reproduced by any combination of the stellar parameters. However, we cannot rule out that these mismatches are artefacts caused by inadequate data reduction.

Obviously these three white dwarfs need to be reobserved before any firm conclusion can be drawn. Therefore we shall exclude these stars from further discussion.

\subsection{Synopsis}

Table 3 summarizes the results from the present study (19 stars) as well as those of Papers I (13 stars) and II (25 stars). However, WD 2329-291, analyzed in Paper II, was shown recently by 

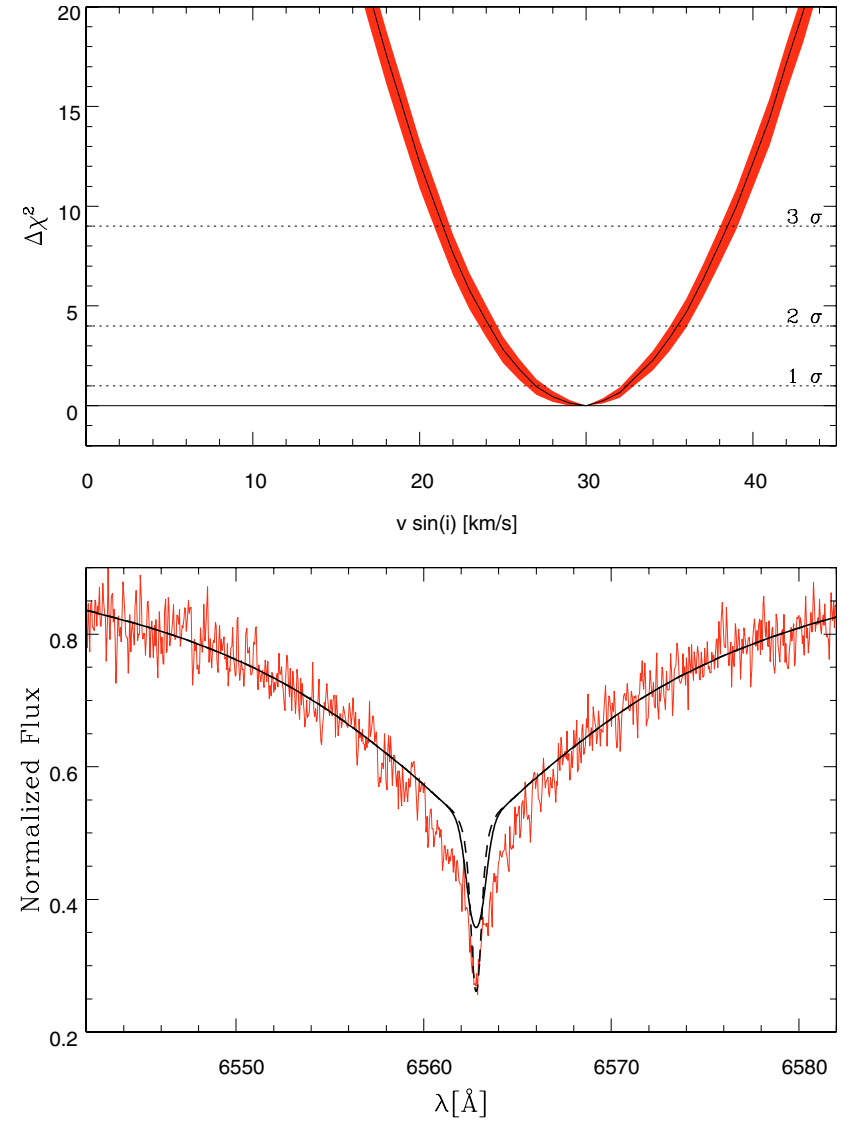

Fig. 6. $\mathrm{H} \alpha$ line profile fit for WD 0628-020, a star with a peculiar $\mathrm{H} \alpha$ line profile. The upper panel displays the run of $\Delta \chi^{2}$ with $v \sin i$ from which the best fit and error limits are read off. The lower panel compares the observed profile to a synthetic line profile calculated without rotation (dashed line) and for the best fit $v \sin i$ (full line). Obviously the observed line profile is not matched by either profiles. While the line core is well matched assuming no rotation at all, the inner wings cannot be matched by any reasonable rotation velocity.

Lisker et al. (2004) to be a sdB star. Therefore we excluded it from further discussion of the remaining 56 white dwarf stars.

We divide the complete sample into a hot one with radiative atmospheres and a cool one with presumedly convective atmospheres and divide the sample with a borderline at $T_{\text {eff }}=$ $14000 \mathrm{~K}$. There are, however, six stars with $T_{\text {eff }}$ close to the borderline, i.e. in the transition zone from radiative to convective atmospheres. Therefore we consider a group of hot white dwarfs consisting of all programme stars with $T_{\text {eff }}=14600 \mathrm{~K}$ (WD 1919+145) or higher and a group of cool white dwarfs with $T_{\text {eff }}=12800 \mathrm{~K}($ WD $0921+354)$ or less.

\section{Hot stars}

For all but three of our 28 stars with $T_{\text {eff }} \geq 14600 \mathrm{~K}$ there is no hint of rotation, with upper limits ranging from $1 \mathrm{~km} \mathrm{~s}^{-1}$ to $24 \mathrm{~km} \mathrm{~s}^{-1}$. However, the line broadening is more likely to be due to magnetic fields in WD 1531-022 (see Paper II). Therefore only two rotating object (WD 0836+201 and WD 2039-682) are found in the hotter temperature

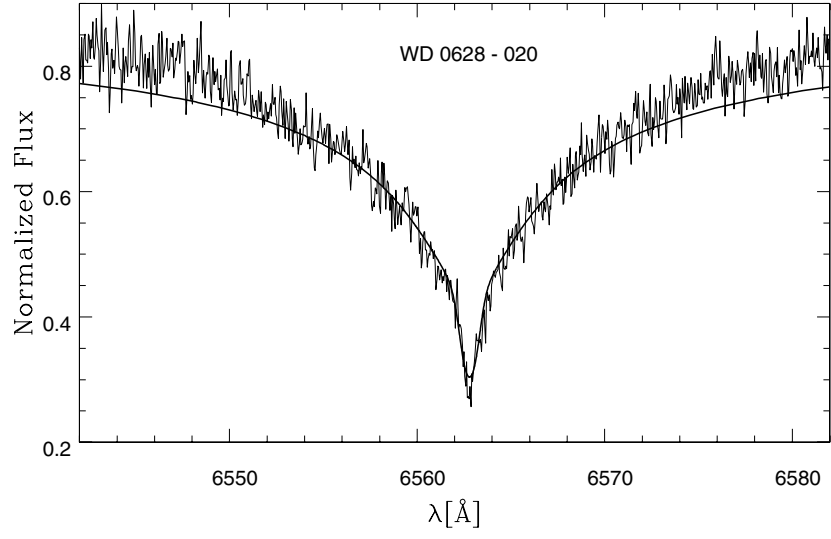

Fig. 7. $\mathrm{H} \alpha$ line profile fit for WD 0628-020. The observed profile is compared to a synthetic line profile calculated at a temperature $1000 \mathrm{~K}$ below the value given in Table 1 and for $v \sin i=20 \mathrm{~km} \mathrm{~s}^{-1}$. While the line core and the inner line wings are matched perfectly, the outer line wings are not reproduced very well.

range, which are amongst the fastest rotating DA stars known (80 $\mathrm{km} \mathrm{s}^{-1}$ and $66 \mathrm{~km} \mathrm{~s}^{-1}$, respectively).

\section{Cool stars}

For lower temperatures we find no hint of extra broadening only in 8 out of 22 stars with upper limits to the projected rotational velocites ranging from $7 \mathrm{~km} \mathrm{~s}^{-1}$ to $35 \mathrm{~km} \mathrm{~s}^{-1}$.

For the remaing stars, extra broadening has to be accounted for. Again the broadening is probably caused by magnetic fields in two cases (WD 1953-011 and WD 2105-820, see Paper II).

ZZ Ceti stars For the four ZZ Ceti variables the line profiles are not well matched by rotational profiles (see Paper II and Fig. 4). Unlike the three ZZ Ceti stars studied in Paper II, WD 0921+354 displays a very narrow line core.

Non-variables There are the remaining eight stars whose $\mathrm{H} \alpha$ line profiles can reasonably well be matched by rotationally broadened synthetic line profiles with projected rotational velocities ranging from $20 \mathrm{~km} \mathrm{~s}^{-1}$ to $72 \mathrm{~km} \mathrm{~s}^{-1}$.

\section{Borderline objects}

No hint of extra broadening can be found for four of the six stars near the borderline $T_{\text {eff }}=14000 \mathrm{~K}$ with upper limits to the projected rotational velocity of $7 \mathrm{~km} \mathrm{~s}^{-1}$ to $35 \mathrm{~km} \mathrm{~s}^{-1}$. Two stars can be matched by assuming rather slow rotation, $v \sin i=$ $23 \mathrm{~km} \mathrm{~s}^{-1}$ and $28 \mathrm{~km} \mathrm{~s}^{-1}$. In the latter case an even lower projected rotational velocity of $13 \mathrm{~km} \mathrm{~s}^{-1}$ was derived in Paper II, but no error could be estimated.

\section{Comparison}

Figure 8 displays the complete sample of stars from Papers I, II and this paper (cf. Table 3) in a $\log g-T_{\text {eff diagram. To }}$ be consistent with Paper II we distinguish between "fast" rotators ( $v \sin i \geq 20 \mathrm{~km} \mathrm{~s}^{-1}$ within error margins) and "slow" (or "non") rotators $\left(v \sin i<20 \mathrm{~km} \mathrm{~s}^{-1}\right.$ ). Also plotted are the 
Table 3. Summary of projected rotational velocities ( $1 \sigma$ detection limit) and stellar parameters of all stars from this paper and Papers I and II.

\begin{tabular}{|c|c|c|c|c|c|c|c|c|c|c|c|}
\hline 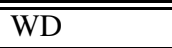 & 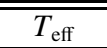 & $\overline{\log g}$ & 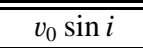 & $\overline{v_{\max } \sin i}$ & Ref. & 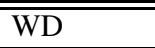 & $\overline{\overline{T_{\text {eff }}}}$ & $\overline{\log g}$ & 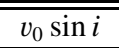 & $\overline{v_{\max } \sin i}$ & Ref. \\
\hline $1710+683$ & 6825 & 7.94 & $72_{-10}^{+11}$ & & III & $1919+145$ & 14600 & 8.09 & & $<6$ & II \\
\hline $0807+484$ & 7000 & 8.01 & & $<18$ & III & $0352+096$ & 14770 & 8.16 & & $<13$ & I \\
\hline $2253-081$ & 7160 & 8.43 & $36_{-7}^{+15}$ & & III & $2007-303$ & 15150 & 7.86 & & $<7$ & II \\
\hline $0251-008$ & 7700 & 8.05 & $24_{-5}^{+4}$ & & III & $0406+169$ & 15190 & 8.30 & & $<20$ & I \\
\hline $1953-011$ & 7950 & 8.41 & $173 \pm 10^{b}$ & & II & $0810+234$ & 15220 & 7.99 & & $<16$ & III \\
\hline $2359-434^{d, e}$ & 8850 & 8.57 & & $<20$ & II & $1143+321$ & 15489 & 7.97 & & $<2$ & I \\
\hline $0518+333^{f}$ & 9140 & 8.30 & & $<7$ & III & 0310-688 & 15700 & 8.16 & & $<4$ & II \\
\hline $1510+566$ & 9280 & 7.98 & & $<11$ & III & $0220+222$ & 15840 & 7.95 & & $<9$ & III \\
\hline $2258+406$ & 9860 & 8.20 & $20_{-5}^{+6}$ & & III & $2039-682$ & 16050 & 8.44 & $78 \pm 6^{b}$ & & II \\
\hline $2115-560$ & 9950 & 8.13 & & $<35$ & II & & & & $82 \pm 5$ & & $\mathrm{M} \& \mathrm{M}$ \\
\hline $0906+296$ & 10000 & 7.86 & $41_{-5}^{+6}$ & & III & $0413-077$ & 16570 & 7.86 & & $<1$ & I \\
\hline $2007-219^{d}$ & 10000 & 8.18 & & $<20$ & II & $0836+201$ & 16630 & 8.04 & $66 \pm 14$ & & III \\
\hline $1840-111^{d}$ & 10230 & 8.18 & & $<20$ & II & $0837+199$ & 17100 & 8.32 & & $<9$ & I \\
\hline $1147+255$ & 10260 & 8.12 & $24 \pm 7$ & & III & $2149+021$ & 17650 & 7.99 & $16 \pm 5$ & $<21$ & II \\
\hline $0030+444$ & 10440 & 8.02 & $27_{-4}^{+5}$ & & III & $0047-524$ & 18750 & 7.83 & $1 \pm 11$ & $<12$ & II \\
\hline $2105-820$ & 10760 & 8.25 & $65^{-4} 5^{b}$ & & II & $1211+392$ & 18800 & 7.70 & & $<18$ & III \\
\hline $1544-377^{d}$ & 11250 & 8.09 & & $<20$ & II & $1531-022$ & 18850 & 8.39 & $50 \pm 6^{b}$ & & II \\
\hline $2326+049^{a}$ & 11600 & 8.05 & $45 \pm 5$ & & II & 2039-202 & 19350 & 7.95 & & $<15$ & II \\
\hline $1422+095^{a}$ & 11950 & 7.87 & $29 \pm 7$ & & II & $1943+163$ & 19400 & 7.80 & $16 \pm 8$ & $<24$ & II \\
\hline $1240+754$ & 12000 & 7.96 & $30_{-10}^{+7}$ & & III & $0421+162$ & 19570 & 8.09 & & $<8$ & I \\
\hline $1425-811^{a}$ & 12200 & 7.99 & $38 \pm 3$ & & II & $2032+248$ & 19900 & 7.87 & $11 \pm 5$ & $<16$ & II \\
\hline $0921+354^{a}$ & 12830 & 7.92 & $36_{-5}^{+7}$ & & III & $0431+125$ & 21340 & 8.04 & $15_{-5}^{+7}$ & & I \\
\hline $0726+393$ & 13600 & 7.76 & & $<7$ & I & $0836+197$ & 21900 & 8.45 & & $<22$ & I \\
\hline $1706+332$ & 13600 & 7.79 & $23_{-4}^{+3}$ & & III & $1620-391$ & 24250 & 8.05 & & $<8$ & II \\
\hline $1716+020$ & 13620 & 7.79 & & $<11$ & III & $0425+168$ & 24420 & 8.11 & & $<18$ & I \\
\hline $1827-106$ & 13800 & 7.61 & & $<20$ & II & $2014-575$ & 26850 & 7.80 & & $<14$ & II \\
\hline $1743-132$ & 13950 & 7.71 & $28_{-4}^{+3 c}$ & & III & $0438+108$ & 27390 & 8.07 & & $<14$ & I \\
\hline \multirow[t]{2}{*}{$1911+135$} & 14040 & 7.83 & & $<2$ & I & $1615-154$ & 29600 & 7.95 & & $<13$ & II \\
\hline & & & & & & $1314+293$ & 49000 & 7.70 & & $<11$ & I \\
\hline $\begin{array}{l}\text { Paper I: Heb } \\
\text { Paper II: Koe } \\
\text { Paper III: thi } \\
\text { M\&M: Maxt } \\
{ }^{a} \text { Known ZZ }\end{array}$ & $\begin{array}{l}\text { et al. (19 } \\
\text { er et al. } \\
\text { Paper. } \\
\text { \& Mars } \\
\text { eti varial }\end{array}$ & $\begin{array}{l}7) . \\
998) . \\
(1999) \\
\text { e star. }\end{array}$ & & & & $\begin{array}{l}{ }^{{ }^{b}} \text { broaden } \\
{ }^{c} v \sin i= \\
{ }^{d} \text { poor fit } \\
{ }^{e} \text { possibly } \\
{ }^{f} \text { probably }\end{array}$ & $\begin{array}{l}g \text { probal } \\
3 \mathrm{~km} \mathrm{~s}^{-1} \\
\text { ee Paper } \\
\text { nagnetic } \\
\text { binary. }\end{array}$ & $\begin{array}{l}\text { y cause } \\
\text { Paper } \\
\text { I). }\end{array}$ & by a mas & etic field. & \\
\hline
\end{tabular}

ZZ Ceti stars and the magnetic white dwarfs, as well as evolutionary tracks by Blöcker (1995) and Driebe et al. (1998).

Comparing the results for the cool DAs with supposedly convective atmospheres to that for the hot DAs with supposedly radiative atmospheres, we find a striking difference. While there is no indication of rotation or any other extra broadening mechanism in the vast majority of the hot stars, we have to call upon extra broadening for the majority of the cool stars, as only in three cases are magnetic fields the likely cause. For the four ZZ Ceti stars rotation can be excluded as the broadening mechanism because that would be inconsistent with the analysis of their pulsation patterns. The $\mathrm{H} \alpha$ lines of the other stars can be reasonably well matched by rotational profiles. Three stars are excluded from this discussion because their $\mathrm{H} \alpha$ line profiles are very peculiar. These objects are amongst the coolest stars in our sample.

\section{Discussion}

We analyzed the $\mathrm{H} \alpha$ line profiles of 22 hydrogen-rich white dwarfs (DA). Synthetic spectra were calculated from LTE model atmospheres by treating the statistical equilibrium of hydrogen in detail in NLTE in order to model the sharp $\mathrm{H} \alpha$ line core reliably. The synthetic spectra were broadened by rotational profiles and a $\chi^{2}$ minimization method was used to determine projected rotational velocities for 19 of our 22 programme stars from high resolution spectra obtained with the HIRES spectrograph at the Keck I telescope. We combined our measurements with data from previous analyses (Heber et al. 1997; Koester et al. 1998) which were based on similar spectra and model atmospheres to derive a homogenous set of $v$ sin i measurements of 56 DA white dwarfs. This sample contains four ZZ Ceti variables and four probably magnetic white dwarfs. In the latter cases the widening of the line core is caused by Zeemann splitting.

As already noted in Paper II the results for hot stars with supposedly radiative atmospheres differ significantly from those for the cooler stars with supposedly convective atmospheres. Our sample contains 28 hot and 22 cool DA stars. Excluding the magnetic white dwarf from the hot sample, it was possible to match the observed profiles with the synthetic spectra well for the remaining 27 stars. Only in two cases was 


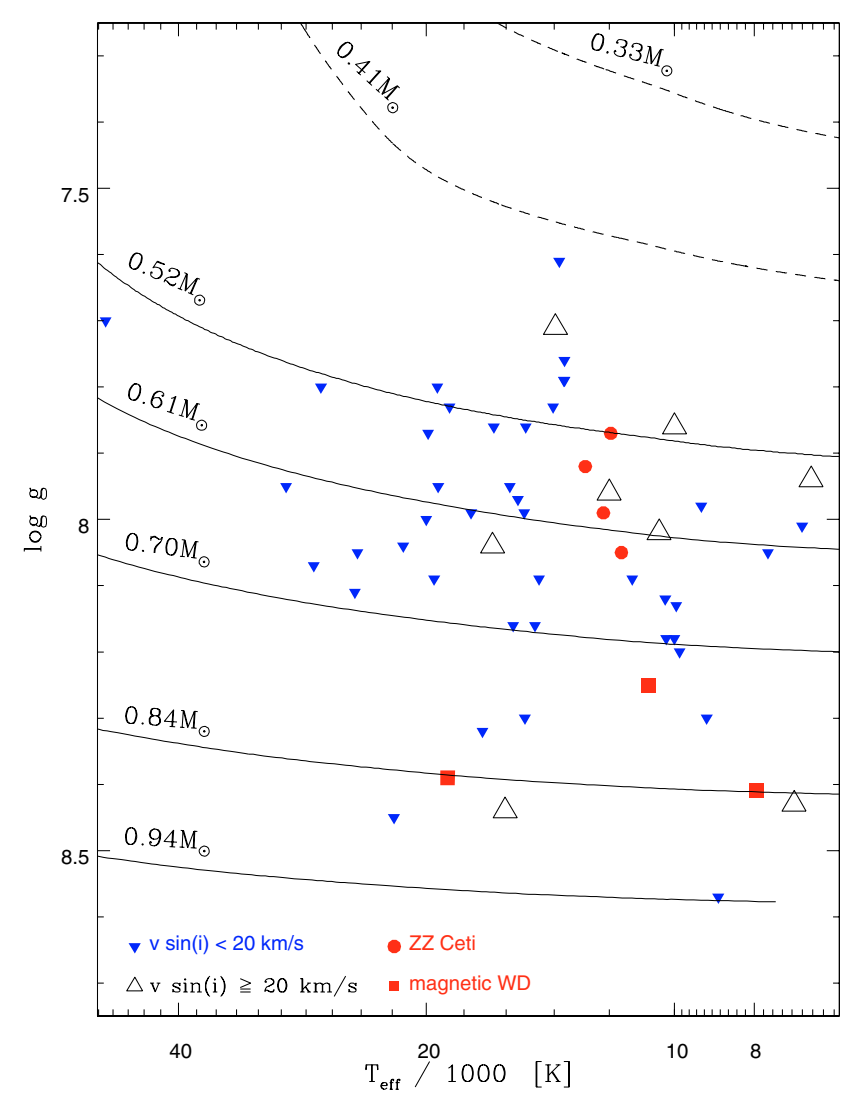

Fig. 8. $T_{\text {eff }}-\log g$ diagram for DA white dwarfs from Papers I and II and this paper. Stars are marked as open triangles if the rotation velocity derived from our analysis exceeds a $20 \mathrm{~km} \mathrm{~s}^{-1}$ limit within the error margins. Non-rotating objects or "slow" rotators are indicated as filled triangles, while open squares marked objects with peculiar line broadening due to magnetic fields. ZZ Ceti stars are displayed as open circles. Also shown are evolutionary tracks for white dwarfs (Blöcker 1995, solid lines; and Driebe et al. 1998, dashed lines) labelled with the stellar masses.

significant rotation detected. For all other hot stars the spectral analysis did not reveal any rotation.

For more than half of the cooler DA stars, however, the $\mathrm{H} \alpha$ profile did show evidence of additional broadening. The spectra of ten cool stars were consistently matched by line profiles with projected rotation velocities between $23 \mathrm{~km} \mathrm{~s}^{-1}$ and $72 \mathrm{~km} \mathrm{~s}^{-1}$. Close inspection of the line profiles revealed significant deviations from the rotational profile for the four ZZ Ceti stars.

All four ZZ ceti stars in our sample show evidence of peculiar line broadening. In principle, the rotation velocity of a ZZ Ceti star can be determined independently by measuring the rotation-induced splitting of the pulsation modes. As noted already in Paper II the low rotation periods $(\approx 1$ day) derived from asteroseismology are at variance with the spectroscopic results. Hence the line broadening cannot be caused by rotation and other broadening mechanisms have been invoked. Lineof-sight variations have indeed be discovered in the ZZ Ceti stars G 29-38 (up to $5 \mathrm{~km} \mathrm{~s}^{-1}$, Van Kerkwijk et al. 2000) and HS 0507+0434B (of $2.6 \mathrm{~km} \mathrm{~s}^{-1}$, Kotak et al. 2002b) and were attributed to horizontal surface motions associated with $g$ mode pulsation. The measured amplitude, however, is too small to account for the line broadening observed in the four ZZ Ceti stars in our sample (36 km s${ }^{-1}$ to $45 \mathrm{~km} \mathrm{~s}^{-1}$ ). Recently, Thompson et al. (2003) reobserved G 29-38 at high spectral resolution and found radial velocity changes as large as $16.5 \mathrm{~km} \mathrm{~s}^{-1}$, but concluded that neither rotation nor pulsation can explain the truncated shape observed for the ZZ Ceti star's $\mathrm{H} \alpha$ line core. Therefore the physical mechanism still remains unclear.

In view of the ZZ Ceti results, it may be premature to conclude that the extra broadening that we see in the non-variable cool DAs is due to rotation. The average rotation velocities in a sample of hot white dwarfs should be the same as in a sample of cool white dwarfs. Moreover, one would expect similar broadening for all stars within a given parameter range. It is very puzzling, for example, that WD $0807+484$ does not show any sign of extra broadening, while WD $1710+683$ - having almost the same $T_{\text {eff }}$ and $\log g$-does. Thus we conjecture that the physics of $\mathrm{H} \alpha$ line formation in convective white dwarf atmospheres is not yet sufficiently well understood. Additional parameters may have to be taken into account. For example, the helium abundance in a convectively mixed atmosphere might be related to the mass of the hydrogen envelope and thus could be different in two stars having otherwise identical parameters.

New high resolution observations of excellent $\mathrm{S} / \mathrm{N}$ for a large sample of both hot and cool white dwarfs are required to gain insight into the rotational properties of white dwarf stars and possible other broadening mechanisms. Recently the ESO Supernova Ia Progenitor Survey (SPY) provided high resolution spectra of more than 1000 white dwarfs (Napiwotzki et al. 2003). Many of these are of sufficiently high $\mathrm{S} / \mathrm{N}$ to allow precise measurements of the $\mathrm{H} \alpha$ lines of hundreds of white dwarfs.

Acknowledgements. C.K. gratefully acknowledges financial support by the Deutsche Forschungsgemeinschaft (grant Na 365-x/2-1). The authors thank the referee for constructive comments and suggestions.

\section{References}

Bergeron, P., Saffer, R. A., \& Liebert, J. 1992, ApJ, 394, 228

Bergeron, P., Liebert, J., \& Fulbright, M. S. 1995a, ApJ, 444, 810

Bergeron, P., Wesemael, F., \& Beauchamp, A. 1995b, PASP, 107, 1047

Bergeron, P., Leggett S. K., \& Ruiz, M. T. 2001, ApJS, 133, 413

Blöcker, T. 1995, A\&A, 299, 755

Bradley, P. A. 1998, ApJS, 116, 307

Claver, C. F., Liebert, J., Bergeron, P., \& Koester, D. 2001, ApJ, 563, 987

Driebe, T., Schönberner, D., Blöcker, T., \& Herwig, F. 1998, A\&A, 339,123

Gray, D. F. 1992, The observation and analysis of stellar photopheres (Cambridge University Press), Cambridge Astrophys. Ser., 20, 374

Giovannini, O., Kepler, S. O., Kanaan, A., et al. 1998, BaltA, 7, 131

Greenstein, J. L., Boksenberg, A., Carswell, R., \& Shortridge, K. 1977, ApJ, 212, 186

Heber, U., Napiwotzki, R., \& Reid, I. N. 1997, A\&A, 323, 819

Kepler, S. O., Giovannini, O., Kanaan, A., Wood, M. A., \& Claver, C. F. 1995, BaltA, 4, 157

Kepler, S. O., Mukadam, A., Winget, D. E., et al. 2000, ApJ, 534, L185

Kidder, K. M., Holberg, J. B., \& Mason, P. A. 1991, AJ, 101, 579 
Koester, D., \& Herrero, A. 1988, A\&A, 332, 910

Koester, D., Dreizler, S., Weidemann, V., \& Allard, N. F. 1998, A\&A, 338,612

Kotak, R., van Kerkwijk, M. H., \& Clemens, J. C. 2002, A\&A, 388, 219

Lisker, T., Heber, U., Napiwotzki, R., et al. 2004, A\&A, submitted Maxted, P. F. L., \& Marsh, T. R. 1999, MNRAS, 307, 122

McCook, G. P., \& Sion, E. M. 1999, ApJS, 121, 1

Napiwotzki, R., Green, P. J., \& Saffer, R. A. 1999, ApJ, 517, 399

Napiwotzki, R., Christlieb, N., Drechsel, H., et al. 2003, Messenger 112,25

O’Brien M. S., Clemens J. C., Kawaler S., \& Dehner B. T. 1996, ApJ, 467, 397

Pilachowski, C. A., \& Milkey, R. W. 1984, PASP, 96, 821
Pilachowski, C. A., \& Milkey, R. W. 1987, PASP, 99, 836

Reid, I. N. 1996, AJ, 111, 2000

Saffer, R. A., Bergeron, P., Koester, D., \& Liebert, J. 1994, ApJ, 432, 351

Silvestri, N. M., Oswalt, T. D., Wood, M. A., et al. 2001, ApJ, 121, 503

Thompson, S. E., Clemens, J. C., van Kerkwijk, M. H., \& Koester, D. 2003, ApJ, 589, 921

Van Kerkwijk, M. H., Clemens, J. C., \& Wu, Y. 2000, MNRAS, 314, 209

Weidemann, V., \& Koester, D. 1984, A\&A, 132, 195

Wickramasinghe, D. T., Ferrario, L. 2000, PASP, 112, 873

Winget, D. E., Nather, R. E., Clemens, J. C., et al. 1991, ApJ, 378, 326

Winget, D. E., Nather, R. E., Clemens, J. C., et al. 1994, ApJ, 430, 839 